\title{
Enhancement of second harmonic generation in a doubly resonant metamaterial
}

\section{AUTHOR(S):}

Kanazawa, Tetsuo; Tamayama, Yasuhiro; Nakanishi, Toshihiro; Kitano, Masao

\section{CITATION:}

Kanazawa, Tetsuo ...[et al]. Enhancement of second harmonic generation in a doubly resonant metamaterial. APPLIED PHYSICS LETTERS 2011, 99(2): 024101.

\section{ISSUE DATE:}

2011-07

URL:

http://hdl.handle.net/2433/160631

\section{RIGHT:}

Copyright 2011 American Institute of Physics. This article may be downloaded for personal use only. Any other use requires prior permission of the author and the American Institute of Physics. The following article appeared in APPLIED PHYSICS LETTERS 99, 024101 (2011) and may be found at http://link.aip.org/link/?apl/99/024101 


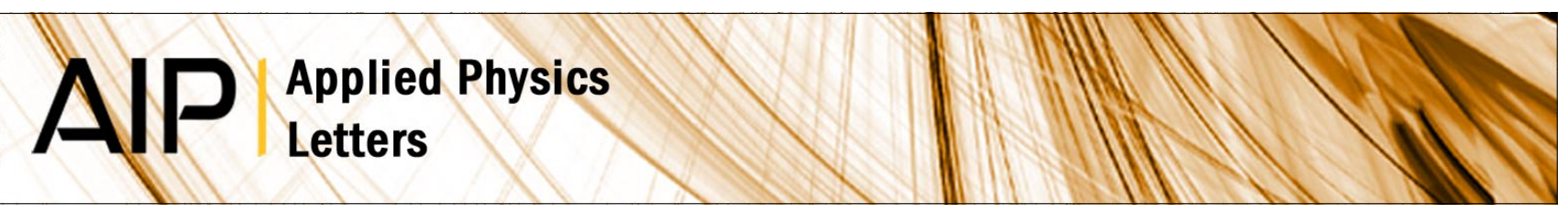

\section{Enhancement of second harmonic generation in a doubly resonant metamaterial}

Tetsuo Kanazawa, Yasuhiro Tamayama, Toshihiro Nakanishi, and Masao Kitano

Citation: Appl. Phys. Lett. 99, 024101 (2011); doi: 10.1063/1.3610471

View online: http://dx.doi.org/10.1063/1.3610471

View Table of Contents: http://apl.aip.org/resource/1/APPLAB/v99/i2

Published by the American Institute of Physics.

\section{Related Articles}

Chalcogenide microwire based Raman laser

Appl. Phys. Lett. 101, 101110 (2012)

Self-accelerating parabolic beams in quadratic nonlinear media

Appl. Phys. Lett. 101, 101109 (2012)

High-order harmonic generation at $4 \mathrm{MHz}$ as a light source for time-of-flight photoemission spectroscopy

Appl. Phys. Lett. 101, 071116 (2012)

Terahertz intracavity generation from output coupler consisting of stacked GaP plates

Appl. Phys. Lett. 101, 021107 (2012)

Analyzing photo-induced interfacial charging in IZO/pentacene/C60/bathocuproine/Al organic solar cells by electric-field-induced optical second-harmonic generation measurement

J. Appl. Phys. 111, 113711 (2012)

\section{Additional information on Appl. Phys. Lett.}

Journal Homepage: http://apl.aip.org/

Journal Information: http://apl.aip.org/about/about_the_journal

Top downloads: http://apl.aip.org/features/most_downloaded

Information for Authors: http://apl.aip.org/authors

\section{ADVERTISEMENT}

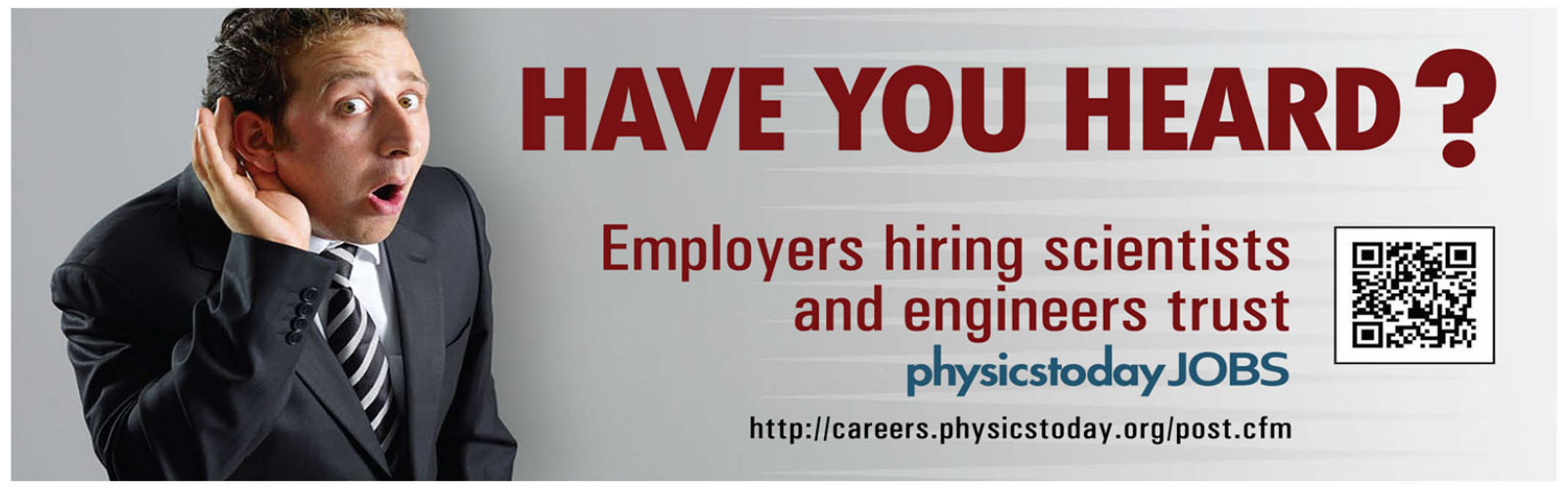




\title{
Enhancement of second harmonic generation in a doubly resonant metamaterial
}

\author{
Tetsuo Kanazawa, Yasuhiro Tamayama, ${ }^{a}$ Toshihiro Nakanishi, and Masao Kitano \\ Department of Electronic Science and Engineering, Kyoto University, Kyoto 615-8510, Japan
}

(Received 4 February 2011; accepted 22 May 2011; published online 12 July 2011)

\begin{abstract}
We investigate second harmonic $(\mathrm{SH})$ generation in a doubly resonant metamaterial. We show that SH generation can be enhanced when the resonant condition is satisfied for the SH frequency as well as for the fundamental frequency. A unit cell of the doubly resonant metamaterial consists of two coupled resonators, one of which resonates at the fundamental frequency, whereas the other resonates around the SH frequency. We observe that the $\mathrm{SH}$ generation in the doubly resonant metamaterial is 4.6 times as large as that in a singly resonant metamaterial. (C) 2011 American Institute of Physics. [doi:10.1063/1.3610471]
\end{abstract}

Metamaterials are arrays of artificial structures having dimensions that are much smaller than the wavelength of electromagnetic waves. Metamaterials are used to realize media with unusual properties that may not be readily available in nature, e.g., negative refractive index media, transformation media, slow light media, and switchable media. ${ }^{1}$ The properties of metamaterials are determined by the shape and material of the constituent, and, therefore, devices with desired functions can be obtained by designing the constituent.

Pendry et al. showed that when a constituent of a metamaterial is a resonant conductive element, electromagnetic energy is squeezed into a small volume of the constituent at the resonant frequency. ${ }^{2}$ They predicted that if a nonlinear material is placed in the volume, the nonlinear behavior of the material is enhanced. Thus far, this phenomenon has been applied to second harmonic ( $\mathrm{SH}$ ) and higher-order harmonic generations, ${ }^{3-7}$ nonlinear tunable metamaterials, ${ }^{5,8,9}$ and bistable media. ${ }^{8}$

In this Letter, we introduce a doubly resonant metamaterial to generate the SH waves more efficiently in the metamaterial than in the singly resonant metamaterial used in the previous studies. ${ }^{3-7}$ We demonstrate that the SH generation can be enhanced if the resonant condition is satisfied for the $\mathrm{SH}$ frequency as well as for the fundamental frequency. First, using an electric circuit model, we show that SH generation can be further enhanced by introducing a resonator for the SH frequency in addition to a resonator for the fundamental frequency. Then, a unit structure of the metamaterial derived from the circuit model is presented. Finally, experimental results for the SH generation using the microwave metamaterial are described. Although the proposed method of enhancing the SH generation is demonstrated in the microwave region, it can also be used in the terahertz and optical regions.

We describe the concept of enhancing the SH generation using equivalent circuit models of resonant metamaterials. A singly resonant metamaterial can be modeled as an inductorcapacitor-resistor series resonant circuit, as shown in Fig. 1(a). The external electromagnetic field corresponds to the voltage source $V \cos \omega t$. The voltage across the capacitor

a)Electronic mail: tama@giga.kuee.kyoto-u.ac.jp. reaches a maximum at the resonant angular frequency $\omega_{0}=1 / \sqrt{L C}$. If the capacitor exhibits nonlinearity, a large nonlinear effect is expected at $\omega_{0}{ }^{2}$ We assume that the voltage across the nonlinear capacitor is written as $v_{C}=q / C(q)=q / C+\alpha q^{2}$, where $q$ is the charge in the nonlinear capacitor and $\alpha$ is the nonlinear coefficient. Using the second-order perturbation method under a weak nonlinearity condition, ${ }^{10}$ the current amplitude oscillating at $2 \omega$ is obtained as

$$
|I(2 \omega)|=\frac{|\alpha| V^{2}}{\omega^{2}\left|Z(2 \omega) Z(\omega)^{2}\right|},
$$

where $Z(\omega)=R-\mathrm{i}[\omega L-1 /(\omega C)]$ is the impedance of the circuit. When $\omega$ is tuned to $\omega_{0},|Z(\omega)|$ takes a minimum value and $|I(2 \omega)|$ is maximized. This implies that the strong SH signal is generated from the singly resonant metamaterial when $\omega=\omega_{0}$. Here, note that $|I(2 \omega)|$ is further enhanced by also reducing $|Z(2 \omega)|$ in some way. That is, more efficient $\mathrm{SH}$ generation from the metamaterial can be achieved when the resonant conditions for both the fundamental and SH frequencies are satisfied.

We introduce a doubly resonant circuit shown in Fig. 1(b) for the enhancement of the SH current. This circuit is composed of two resonant circuits: the primary circuit with resonant angular frequency $\omega_{1}=1 / \sqrt{L_{1} C_{1}}$ and the secondary circuit with resonant angular frequency $\omega_{2}=1$ / $\sqrt{L_{2} C_{2}} \simeq 2 \omega_{1}$. These resonators are coupled via a mutual inductance $M$. Kirchhoff's voltage law yields the coupled differential equations:

$$
\begin{gathered}
L_{1} \frac{\mathrm{d}^{2} q_{1}}{\mathrm{~d} t^{2}}+R_{1} \frac{\mathrm{d} q_{1}}{\mathrm{~d} t}+\frac{q_{1}}{C_{1}}+\alpha q_{1}^{2}-M \frac{\mathrm{d}^{2} q_{2}}{\mathrm{~d} t^{2}}=V \cos \omega t, \\
L_{2} \frac{\mathrm{d}^{2} q_{2}}{\mathrm{~d} t^{2}}+R_{2} \frac{\mathrm{d} q_{2}}{\mathrm{~d} t}+\frac{q_{2}}{C_{2}}-M \frac{\mathrm{d}^{2} q_{1}}{\mathrm{~d} t^{2}}=0 .
\end{gathered}
$$

Here, the voltage across the nonlinear capacitor is expanded up to the second order of the charge $q_{1}$. Applying the secondorder perturbation method to Eqs. (2) and (3), we obtain 

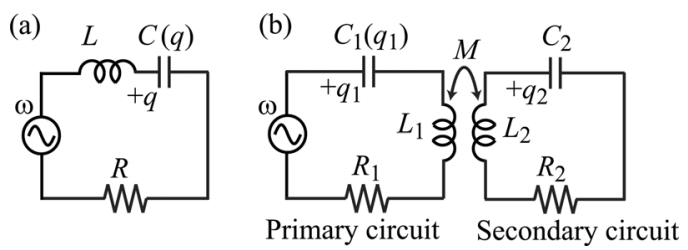

FIG. 1. (a) Singly resonant circuit and (b) proposed doubly resonant circuit for enhancing the SH current. The circuit constants satisfy $1 / \sqrt{L_{1} C_{1}}=\omega_{1}$ and $1 / \sqrt{L_{2} C_{2}}=\omega_{2} \simeq 2 \omega_{1}$.

$$
\begin{gathered}
\left|I_{1}(2 \omega)\right|=\frac{|\alpha| V^{2}}{\omega^{2}\left|Z^{\prime}(2 \omega) Z^{\prime}(\omega)^{2}\right|}, \\
\left|I_{2}(2 \omega)\right|=\frac{|\alpha M| V^{2}}{\omega\left|Z_{2}(2 \omega) Z^{\prime}(2 \omega) Z^{\prime}(\omega)^{2}\right|},
\end{gathered}
$$

where $Z_{1}(\omega)=R_{1}-\mathrm{i}\left[\omega L_{1}-1 /\left(\omega C_{1}\right)\right]$ is the impedance of the primary circuit, $Z_{2}(\omega)=R_{2}-\mathrm{i}\left[\omega L_{2}-1 /\left(\omega C_{2}\right)\right]$ is the impedance of the secondary circuit, and $Z^{\prime}(\omega)=Z_{1}(\omega)$ $+\omega^{2} M^{2} / Z_{2}(\omega)$. Equation (5) shows that, in order to maximize the SH current, $\omega_{2}$ must be shifted appropriately from $2 \omega_{1}$, considering $M$ and the finiteness of the quality factors. Using a weak-coupling approximation, i.e., $\left|Z_{1}(\omega) Z_{2}(\omega)\right| \gg \omega^{2} M^{2}$ and $\left|Z_{1}(2 \omega) Z_{2}(2 \omega)\right| \gg 4 \omega^{2} M^{2}$, Eqs. (4) and (5) can be simplified as follows:

$$
\begin{gathered}
\left|I_{1}(2 \omega)\right| \approx \frac{|\alpha| V^{2}}{\omega^{2}\left|Z_{1}(2 \omega) Z_{1}(\omega)^{2}\right|}, \\
\left|I_{2}(2 \omega)\right| \approx \frac{|\alpha M| V^{2}}{\omega\left|Z_{1}(2 \omega) Z_{2}(2 \omega) Z_{1}(\omega)^{2}\right|} .
\end{gathered}
$$

The SH current flowing in the primary circuit is independent of $M$ and identical to that in the case of the singly resonant circuit. The SH current in the secondary circuit is proportional to $M$. We define the current ratio as $\beta \equiv\left|I_{2}(2 \omega)\right|$ $I_{1}(2 \omega)|\approx| \omega M / Z_{2}(2 \omega) \mid$. When $\beta \gg 1$, the $\mathrm{SH}$ radiation power can be enhanced approximately by a factor of $\beta^{2}$ compared with the case of the singly resonant metamaterial.

Figure 2(a) illustrates a unit cell of a doubly resonant metamaterial derived from the doubly resonant circuit. The primary resonator shown in Fig. 2(b) was fabricated on an FR-4 glass-epoxy printed circuit board of thickness $1.6 \mathrm{~mm}$ with a $35 \mu$ m-thick copper layer. A Schottky diode (Rohm RB886G) was loaded at the center of the I-shaped structure and used as a nonlinear element as in the previous studies. ${ }^{5,7}$ The periodic cascade of structures can be regarded as a series inductor-capacitor resonant circuit. ${ }^{11}$ The inductance is provided by a metal strip in the $x$ direction and the total capacitance is determined by the Schottky diode and gaps between the neighboring structures in the $x$ direction. The primary resonator resonates at $3.4 \mathrm{GHz}$. The secondary resonator shown in Fig. 2(c) was made of a $35 \mu$ m-thick copper film on a polyphenylene ether (PPE) substrate having a thickness of $0.8 \mathrm{~mm}$. We used the PPE substrate, which has a smaller dielectric loss than the FR-4 substrate, to reduce the loss in the secondary resonator, i.e., to achieve the large enhancement factor of the SH generation. The secondary res- (a)

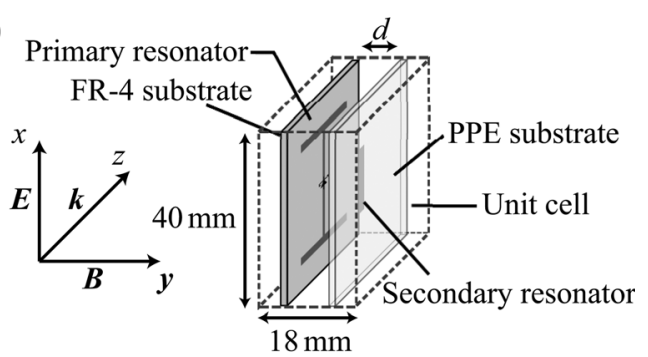

(b)

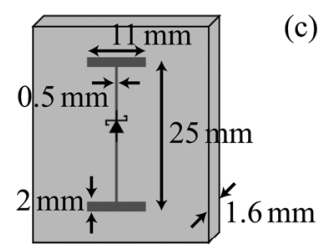

(c)

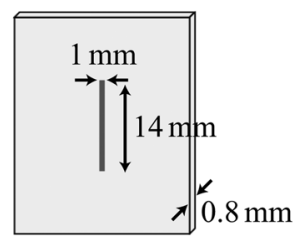

FIG. 2. (a) Unit cell of doubly resonant metamaterial. The cells are aligned periodically in the $x$ and $y$ directions. Geometries of (b) the primary resonator and (c) the secondary resonator.

onator was designed to resonate at $7.5 \mathrm{GHz}$ considering $M$ and the quality factors. The two resonators were separated from each other by distance $d$. Although the $x$-polarized incident electric field can directly excite both the primary and secondary resonators, the current excited in the secondary resonator is negligibly small due to off-resonant excitation. Therefore, the metamaterial can be modeled as the doubly resonant circuit shown in Fig. 1(b).

For the experimental demonstration of the enhancement of the SH generation, we performed transmission measurements of the doubly resonant metamaterial. The mono-layer doubly resonant metamaterial was placed in a parallel plate waveguide composed of two copper plates parallel to the $y z$ plane with a separation of $40 \mathrm{~mm}$. The copper plates are equivalent to periodic boundaries because the electromagnetic fields are uniform in the $x$ direction. A pair of ultrawide band dipole antennas ${ }^{12}$ was used as a transmitter and a receiver. The transmitting antenna was excited by a signal generator and the receiving antenna was connected to a spectrum analyzer.

Figure 3 shows the measured power of the SH wave generated from the doubly resonant metamaterial for three different distances $d$. The $\mathrm{SH}$ power generated from the metamaterial without the secondary resonator is also shown by the solid line. In the absence of the secondary resonator, the radiated SH power reaches a peak around the

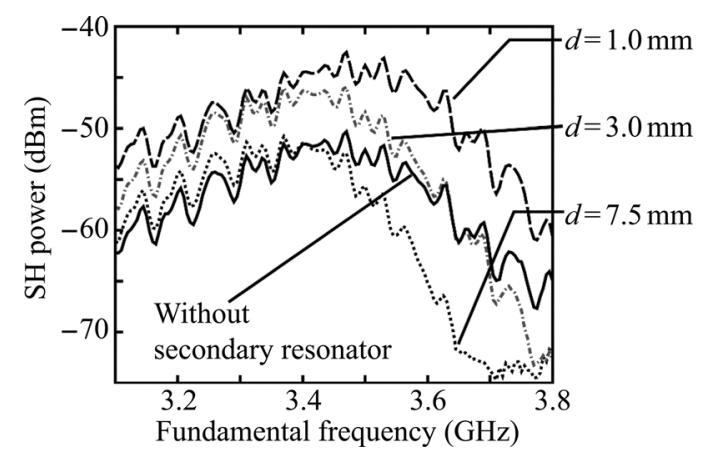

FIG. 3. Measured SH power radiated from the doubly resonant metamaterial for three different $d$ and that from the metamaterial without the secondary resonator as a function of the fundamental frequency. 
fundamental frequency of $3.4 \mathrm{GHz}$, which is the resonant frequency of the primary resonator. Focusing on the SH signal around the fundamental frequency $3.4 \mathrm{GHz}$, the observed $\mathrm{SH}$ power increases with decreasing $d$. The radiated $\mathrm{SH}$ power for $d=1.0 \mathrm{~mm}$ is 4.6 times as large as that in the case of the metamaterial without the secondary resonator. The reason the doubly resonant metamaterial with large $d$ radiates less $\mathrm{SH}$ power than the singly resonant metamaterial will be explained later.

We compare the experimental results with the circuit analysis. We assume that the SH generation from the secondary resonator is much larger than that from the primary resonator. Based on Eq. (7), the total $\mathrm{SH}$ radiation power is written as $P(2 \omega)=a M^{2} \propto|I(2 \omega)|^{2}$, where $a$ is a proportional constant. The mutual inductance $M$ between the primary and secondary resonators can be derived from Neumann's law ${ }^{13}$ and approximated as $M(d) \simeq\left(\mu_{0} / 4 \pi\right)$ $\left(l_{1}+l_{2}\right) \log \left[\left(l_{1}+l_{2}\right) / d\right]$, where $\mu_{0}$ is the permeability in a vacuum and $l_{1}\left(l_{2}\right)$ is the effective length in the $x$ direction of the primary (secondary) resonator. We have assumed that the resonators are infinitely thin conducting wires, that the current distribution is uniform along the $x$ direction, and $l_{1}, l_{2} \gg d$. We fitted the dependence of the measured $\mathrm{SH}$ power on $d$ to $a M^{2}$ in the range of $d=2.5 \mathrm{~mm}-6.5 \mathrm{~mm}$ by varying the parameters $l_{1}+l_{2}$ and $a$.

Figure 4 shows the variation of the $\mathrm{SH}$ power for various $d$. The experimentally obtained $\mathrm{SH}$ power and the fitted curve agree well with each other for $2.5 \mathrm{~mm} \leq d \leq 6.5 \mathrm{~mm}$. Thus, we conclude that the $\mathrm{SH}$ generation is resonantly enhanced in the secondary resonator coupled with the primary resonator via the mutual inductance. The reason the agreement of the measured values with the fitted curve is poor for $d<2.5 \mathrm{~mm}$ or $d>6.5 \mathrm{~mm}$ is as follows. When $d<2.5 \mathrm{~mm}, M$ becomes relatively large and the weak-coupling approximation used in deriving Eq. (7) is not applicable. In the case of $d>6.5 \mathrm{~mm}$, the $\mathrm{SH}$ wave radiated from the secondary resonator is not large enough to neglect that from the primary resonator.

For large $d$, the doubly resonant metamaterial radiates less $\mathrm{SH}$ power than that from the singly resonant metamaterial at around the fundamental frequency of $3.7 \mathrm{GHz}$. In this case, the $\mathrm{SH}$ power is radiated primarily from the primary resonator. $\left|I_{1}(2 \omega)\right|$ in Eq. (4) becomes smaller than $|I(2 \omega)|$ in Eq. (1) because $\left|Z^{\prime}(2 \omega)\right|>|Z(2 \omega)|$. Thus, the total radiated $\mathrm{SH}$ power is smaller than that from the singly resonant metamaterial.

We investigated the SH generation in the doubly resonant metamaterial. Using the electric circuit model, we showed that the efficiency of the SH generation can be enhanced when the resonant conditions for both the SH frequency and the fundamental frequency are satisfied. We measured the power of the radiated $\mathrm{SH}$ wave from the microwave metamaterial and verified that the efficiency of the SH generation was further enhanced by introducing the secondary resonator due to the resonance effect for the $\mathrm{SH}$ frequency.

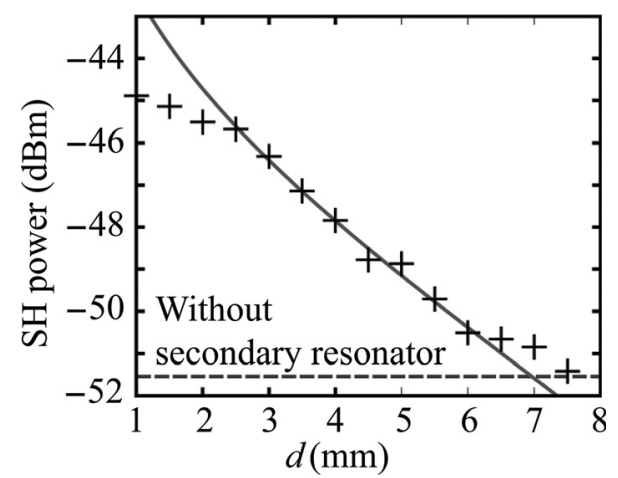

FIG. 4. Measured (cross) and fitted (solid curve) SH powers versus $d$ at the fundamental frequency $3.4 \mathrm{GHz}$. The dashed line represents the $\mathrm{SH}$ power in the absence of the secondary resonator.

The enhancement factor can be increased by reducing the losses in the secondary resonator, increasing the coupling between the two resonators, and finely tuning the resonant frequency of the secondary resonator; therefore, we expect larger $\mathrm{SH}$ radiation by optimizing the structure of the metamaterial. The current method can be applied to other frequency-conversion processes, such as higher-order harmonic generations and sum-frequency mixing. When using the proposed method in a multi-layered metamaterial, it is necessary to design the metamaterial so that the phase-matching condition is also satisfied. ${ }^{14}$

The present research was supported in part by Grants-inAid for Scientific Research Nos. 22109004 and 22560041, by the Global COE program "Photonics and Electronics Science and Engineering" of Kyoto University, and by a research granted from The Murata Science Foundation. One of the authors (Y.T.) would like to acknowledge the support of a Research Fellowship of the Japan Society for the Promotion of Science for Young Scientists.

${ }^{1}$ N. I. Zheludev, Science 328, 582 (2010).

${ }^{2}$ J. B. Pendry, A. J. Holden, D. J. Robbins, and W. J. Stewart, IEEE Trans. Microwave Theory Tech. 47, 2075 (1999).

${ }^{3}$ M. W. Klein, C. Enkrich, M. Wegener, and S. Linden, Science 313, 502 (2006).

${ }^{4}$ M. W. Klein, M. Wegener, N. Feth, and S. Linden, Opt. Express 15, 5238 (2007).

${ }^{5}$ I. V. Shadrivov, A. B. Kozyrev, D. W. van der Weide, and Y. S. Kivshar, Appl. Phys. Lett. 93, 161903 (2008).

${ }^{6}$ E. Kim, F. Wang, W. Wu, Z. Yu, and Y. R. Shen, Phys. Rev. B 78, 113102 (2008).

${ }^{7}$ Z. Wang, Y. Luo, L. Peng, J. Huangfu, T. Jiang, D. Wang, H. Chen, and L. Ran, Appl. Phys. Lett. 94, 134102 (2009).

${ }^{8}$ B. Wang, J. Zhou, T. Koschny, and C. M. Soukoulis, Opt. Express 16, 16058 (2008).

${ }^{9}$ D. A. Powell, I. V. Shadrivov, and Y. S. Kivshar, Appl. Phys. Lett. 95, 084102 (2009).

${ }^{10}$ E. Poutrina, D. Huang, and D. R. Smith, New J. Phys. 12, 093010 (2010).

${ }^{11}$ R. W. Ziolkowski, IEEE Trans. Antennas Propag. 51, 1516 (2003).

${ }^{12}$ E. Lule, T. Babi, and K. Siwiak, Microwave Opt. Technol. Lett. 46, 536 (2005).

${ }^{13}$ J. A. Stratton, Electromagnetic Theory (McGrow-Hill, New York, NY, 1941).

${ }^{14}$ M. V. Gorkunov, I. V. Shadrivov, and Y. S. Kivshar, Appl. Phys. Lett. 88, 071912 (2006). 